\title{
The exploitation of thin film coatings for fibre sensors for the application of chemical sensing
}

T. Allsop, R. Neal, K. Kalli ,E. Davies, S. Rehman, R. R.J. Maier, J. Barton, D.J. Webb, J.D. Jones

I. Bennion

T.Allsop

t.d.p.allsop@aston.ac.uk, Photonics Research Group, Aston University, Aston Triangle, Birmingham, B4 7ET, UK.

R. Neal

R.Neal@plymouth.ac.uk, Dept of Communications and Electrical Engineering, Facility of Technology, University of Plymouth, Plymouth, PL4 8AA, U.K.

K. Kalli

kkalli@cytanet.com.cy, Cyprus University of Technology, Department of Electrical Engineering and Information Technology, 31 Archbishop Kyprianos, Lemessos 3036, Cyprus

E. M. Davies, daviesem@aston.ac.uk, Photonics Research Group, Aston University, Aston Triangle, Birmingham, B4 7ET, UK.

S. Rehman

s.s.rehman@usa.net, STR Fiber Technologies, United Kingdom.

R. R. J. Maier,

R.R.J.Maier@hw.ac.uk, Heriot Watt University, School of EPS, Edinburgh EH14 4AS, UK

J. Barton

j.s.barton@hw.ac.uk, Heriot Watt University, School of EPS, Edinburgh EH14 4AS, UK

J.D. Jones

j.jones@hw.ac.uk, Heriot Watt University, School of EPS, Edinburgh EH14 4AS, UK

D.J. Webb

d.j.webb@aston.ac.uk, Photonics Research Group, Aston University, Aston Triangle, Birmingham, B4 7ET, UK.

I. Bennion

i.bennion@aston.ac.uk, Photonics Research Group, Aston University, Aston Triangle, Birmingham, B4 7ET, UK.

\begin{abstract}
We report on the use of thin film coatings, both single and multi-layered, deposited on the flat side of a lapped, D-shaped fibre to enhance the sensitivity of two kinds of surface plasmon resonance based optical fibre sensors. The first kind involves the use of a tilted Bragg grating inscribed within the fibre core, prior to fibre coating, while the second relies on a surface relief grating photoinscribed after the fibre has been coated. Some of the devices operate in air with high coupling efficiency in excess of $40 \mathrm{~dB}$ and an estimated index sensitivity of $\Delta \lambda / \Delta \mathrm{n}=90 \mathrm{~nm}$ from 1 to 1.15 index range showing potential for gas sensing. Other sensors produced index sensitivities $(\Delta \lambda / \Delta \mathrm{n})$ ranging from $6790 \mathrm{~nm}$ to $12500 \mathrm{~nm}$ in the aqueous index regime. The materials used for these fibre optical devices are germanium, silica, silver, gold and palladium.
\end{abstract}

Keywords: Index Sensing, Coatings, gratings, surface plasmon resonances

Photonics in the Transportation Industry: Auto to Aerospace II, edited by Alex A. Kazemi, Bernard C. Kress Proc. of SPIE Vol. 7314, 73140F · (C) 2009 SPIE · CCC code: 0277-786X/09/\$18 · doi: 10.1117/12.821112 


\section{INTRODUCTION}

There has been a strong interest in recent years in using gratings in fibres to produce chemical sensors, including long period gratings (LPGs) [1, 2, 3], fibre Bragg gratings (FBGs) and tilted fibre Bragg gratings (TFBGs). At present the majority of these sensors are used to detect the heavier organic/inorganic compounds, such as detection of organic aromatic compounds in paraffin [3]. Whilst the index of sensitivity of most grating based devices is highest for solutions that have an index of approximately 1.44, which is good for various organic hydrocarbon compounds, there is a need to increase sensitivity of these devices to aqueous solutions for biochemical applications, where index values typically range from 1.333 to 1.380 (aqueous index regime); for example, the effective index of a cell is about 1.36 to $1.38[4,5]$. Such sensors would also be appropriate for the detection of the lighter hydrocarbon compounds used for Aviation fuel [6] and if their response could be enhanced at even lower refractive indices, for gas detection. Another type of sensor based upon surface plasmon resonance (SPR) is showing the potential to yield high index sensitivities. Surface plasmon resonance is an important optical phenomenon that involves a resonant transfer of the incident light energy to a surface-plasmon (SP) mode in the form of collective electron oscillations in a metal [7]. The plasmons exist at a metaldielectric interface and obey the following dispersion relation for two homogeneous semi-infinite media [8]:

$$
\beta=k \sqrt{\left(\frac{\varepsilon_{m} \cdot n_{s}{ }^{2}}{\varepsilon_{m}+n_{s}{ }^{2}}\right)}
$$

where $\mathrm{k}$ is the free space wave number, $\varepsilon_{\mathrm{m}}$ is the dielectric constant of the metal $\left(\varepsilon_{\mathrm{m}}=\varepsilon_{\mathrm{mr}}+\mathrm{i} \varepsilon_{\mathrm{mi}}\right)$ and $\mathrm{n}_{\mathrm{s}}$ is the refractive index of the dielectric.

The need for high sensitivity at low indices is being addressed with the use of tilted Bragg gratings to assist in the generation of surface plasmon resonances in a fibre configuration $[9,10]$. There is very little published with regards to multi-layered thin film SPR fibre devices, and the majority of these papers, such as [11] and [12], address the SP's optical properties and not their sensing potential.

In this paper, we report on two novel SPR fibre devices whose spectral sensitivities can be modified by using various coatings, in either single or multiple layers. A general observation of these devices is that they have polarisation dependence; the spectral location of maximum coupling to the SPR is highly dependent upon the polarisation state of the illuminating light and can be tuned from $1100 \mathrm{~nm}$ to $1700 \mathrm{~nm}$.

Firstly, we describe a fibre device utilising a TFBG to enhance the coupling of the illuminating light to a SP generated on a coated, lapped single mode fibre $[9,10]$. It was observed that the spectral location of maximum coupling to the SPR was highly dependent upon the polarisation state of the illuminating light and could be tuned from $1100 \mathrm{~nm}$ to $1700 \mathrm{~nm}$, thus producing various SP probe depths, dependent upon the polarisation. It was found that for a device that is still to be fully optimised, the maximum spectral index sensitivity ( $\mathrm{d} \lambda / \mathrm{dn}$ ) was $3365 \mathrm{~nm}$ for the index range 1.335 to 1.370 with coupling strength in excess of $25 \mathrm{dBs}$.

Secondly, we describe a fibre device based upon a surface relief grating-type structure inscribed with UV light into a multi-layered thin film deposited on the flat side of a lapped, D-shaped fibre. The single layered devices were fabricated from germanium, while the multilayered ones comprised layers of germanium, silica, silver and gold. Some of the devices operated in air with high coupling efficiency in excess of $40 \mathrm{~dB}$ and an estimated index sensitivity of $\Delta \lambda / \Delta \mathrm{n}=$ $90 \mathrm{~nm}$ from 1 to 1.15 index range while others provided an index sensitivity of $\Delta \lambda / \Delta \mathrm{n}=12500 \mathrm{~nm}$ for refractive indices from 1.33 to 1.39 .

\section{FABRICATION AND CHARACTERISATION}

The fabrication of the first type of SPR fibre device begins with the inscription of a grating in a UV photosensitive single mode fibre (hydrogenated standard telecommunications fibre) using a uniform phase mask (mask period $=1.0157 \mu \mathrm{m})$ mounted on a goniometer, tilted to a specific angle. Labels are added to indicate the orientation of the tilted grating. Next, the fibre is lapped down to $10 \mu \mathrm{m}$ from the core-cladding interface; it has been estimated that the error associated with this lapping is approximately $\pm 1 \mu \mathrm{m}$. The labels on the fibre are used to indicate which region of cladding is to be removed so that the grating vector, the fibre axis and the normal to the lapped surface all lie in a plane. Thirdly, the flat of the lapped fibre is then coated with either gold or silver layer with a thickness of $38 \mathrm{~nm}$ and $35 \mathrm{~nm}$ respectively, using a sputter machine and mask.

The second type of SPR device is fabricated by firstly mechanically lapping a standard SMF fibre down to $10 \mu \mathrm{m}$ from the core-cladding interface. Secondly, using RF sputtering, a series of coatings are deposited upon the flat of 
the lapped fibre. These coatings consisted of various numbers of layers and materials used such as germanium, silicon dioxide, silver and gold as described below. Thirdly, the coated fibre was exposed to a UV light interference pattern produced by a uniform phase mask with period $1.018 \mu \mathrm{m}$ through laser beam scanning and multi-exposure. This produced a surface relief structure which has dominant spatial periods of $\sim 0.5 \mu \mathrm{m}$ and $\sim 1 \mu \mathrm{m}$. A series of fibre devices were investigated that consisted of various coatings with the two coupling mechanism that produce the surface plasmon resonances, see table 1 .

Table 1 Materials and their thickness used in the fabrication of SPR fibre devices

\begin{tabular}{|c|c|c|c|c|c|}
\hline \multirow{2}{*}{ Sensor Type } & Materials used & $1^{\text {st }}$ layer & $2^{\text {nd }}$ layer & \multicolumn{2}{|c|}{$3^{\text {rd }}$ layer } \\
\cline { 3 - 6 } & $\begin{array}{c}\text { in the } \\
\text { construction }\end{array}$ & $\begin{array}{c}\text { Germanium } \\
\mathrm{nm}\end{array}$ & $\begin{array}{c}\text { Silica } \\
\mathrm{nm}\end{array}$ & $\begin{array}{c}\text { Silver } \\
\mathrm{nm}\end{array}$ & $\begin{array}{c}\text { Gold } \\
\mathrm{nm}\end{array}$ \\
\hline UV grating inscribed & Device Ag Tilted & - & - & 35 & - \\
in the fibre core & Device Au Tilted & - & - & - & 38 \\
& Device Au Tilted & 48 & 48 & - & - \\
\hline \multirow{3}{*}{ UV post processed } & Device Ge overlay & 48 & - & - & - \\
coating & Device SiO overlay & 48 & 48 & 32 & - \\
& Device Ag overlay & 48 & 48 & 32 \\
\hline
\end{tabular}

The rationale for using these materials is in two parts. The first concerns the optical constants of the materials and the requirement that their dispersion relationships must allow coupling to surface plasmons at a metal-dielectric or semiconductor-dielectric interface; both $\mathrm{Ge}$ and $\mathrm{Ag}$ exhibit this behaviour. Secondly, $\mathrm{Ge}$ and $\mathrm{SiO}_{2}$ layers are used due to the fact that it is known from studies of grating formation [12] that when exposed to UV light, Ge/GeO produces photobleaching and compaction of the material, thus producing a surface corrugation on the multi-layered structure.

Prior to UV processing the polarisation dependence had been investigated and a small variation in the overall transmitted optical power with polarisation was found. After UV exposure the devices were further characterised by observing the spectrum of the transmitted light as the azimuth of the polarisation state was changed. These devices now showed a significant variation with polarisation, which is discussed in reference 10. To do this characterisation, light from a broadband light source was passed through a polariser and a polarisation controller before illumination of the sample, with the transmission spectra being monitored using an optical spectrum analyser (accuracy of $0.005 \mathrm{~nm}$ ). The change in polarisation of the illuminating light was monitored with a polarimeter (Tektronix, PAT 9000B) via a polarisation maintaining coupler, see figure 1 . 


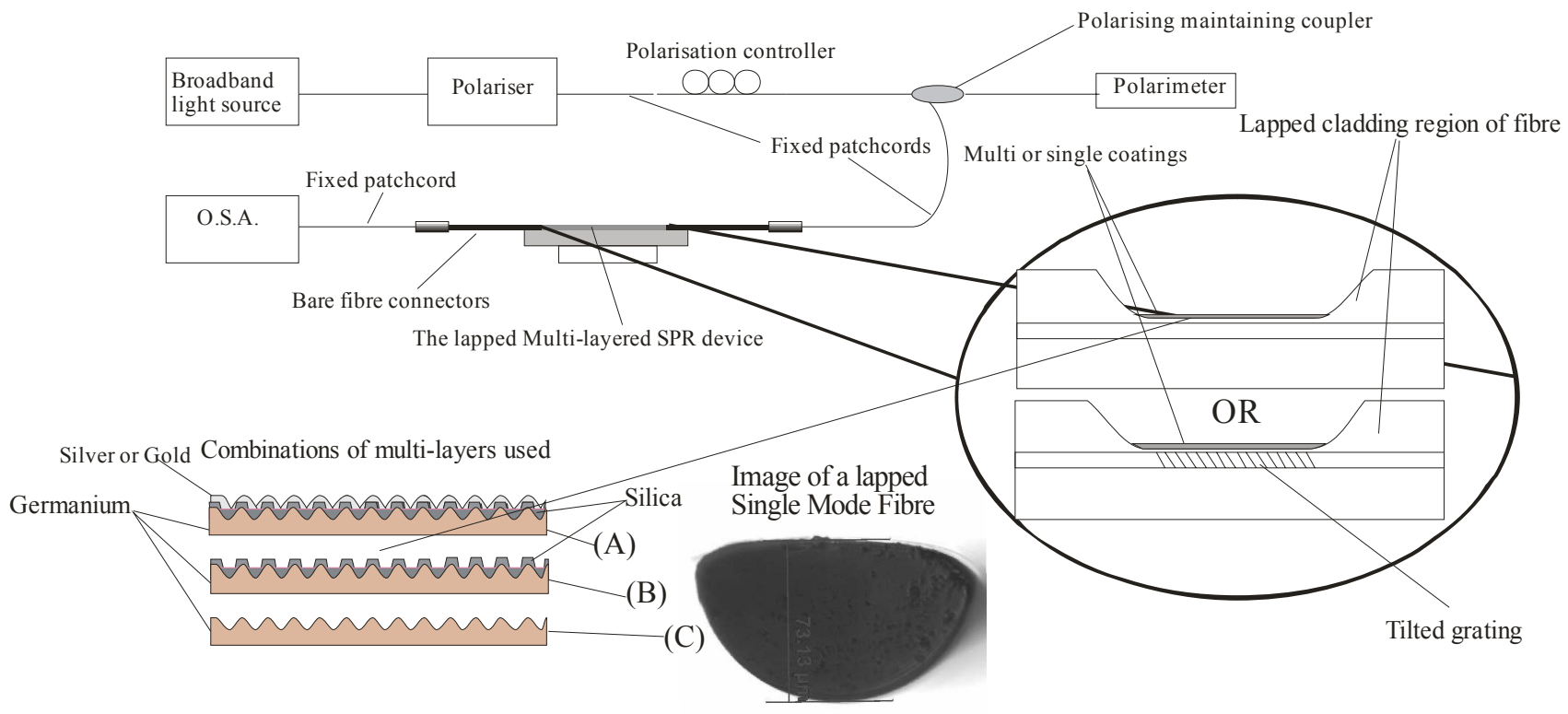

Figure 1. Scheme used for the characterisation of the lapped and multi or single-layer coated fibre devices; typical examples of

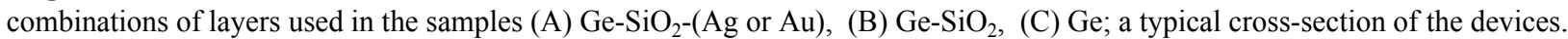

All the fibre devices showed polarisation dependence but the spectral responses differed between the two types of devices. Firstly, the tilted Bragg grating devices could be tuned and produced large extinction ratios over a very wide wavelength range; this occurred for either single or multi-layered coatings, see figure 2.
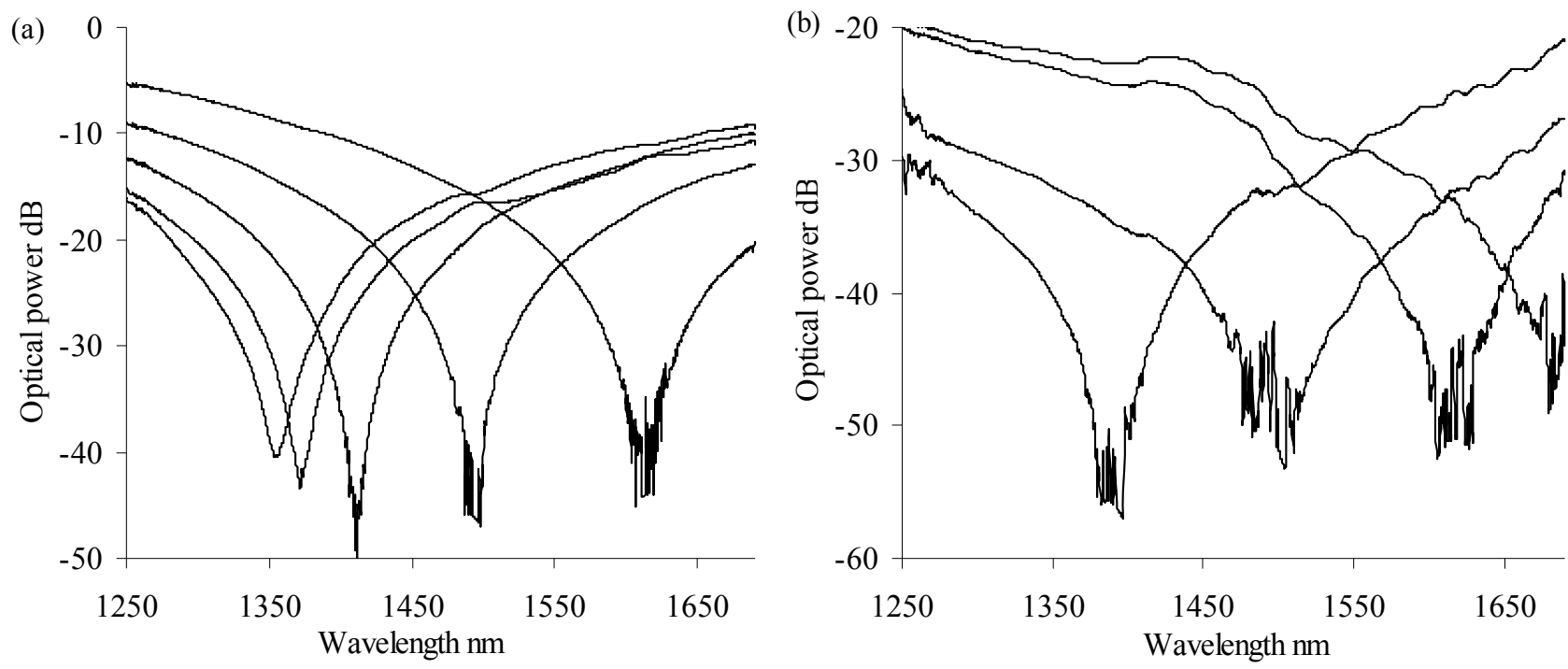

Figure 2 Evidence of surface plasmon resonances obtained at different wavelengths by varying the polarisation of the illuminating light on one device, (a) Au-TFBG, (b) Au-SiO${ }_{2}-\mathrm{Ge}-\mathrm{TFBG}$. Both devices had a 7 degree tilt angle and were submerged in a medium with a refractive index of 1.36 .

Varying the polarisation for the surface relief type SPR fibre devices produced two resonances over the observed wavelength range, see figure 3 . 

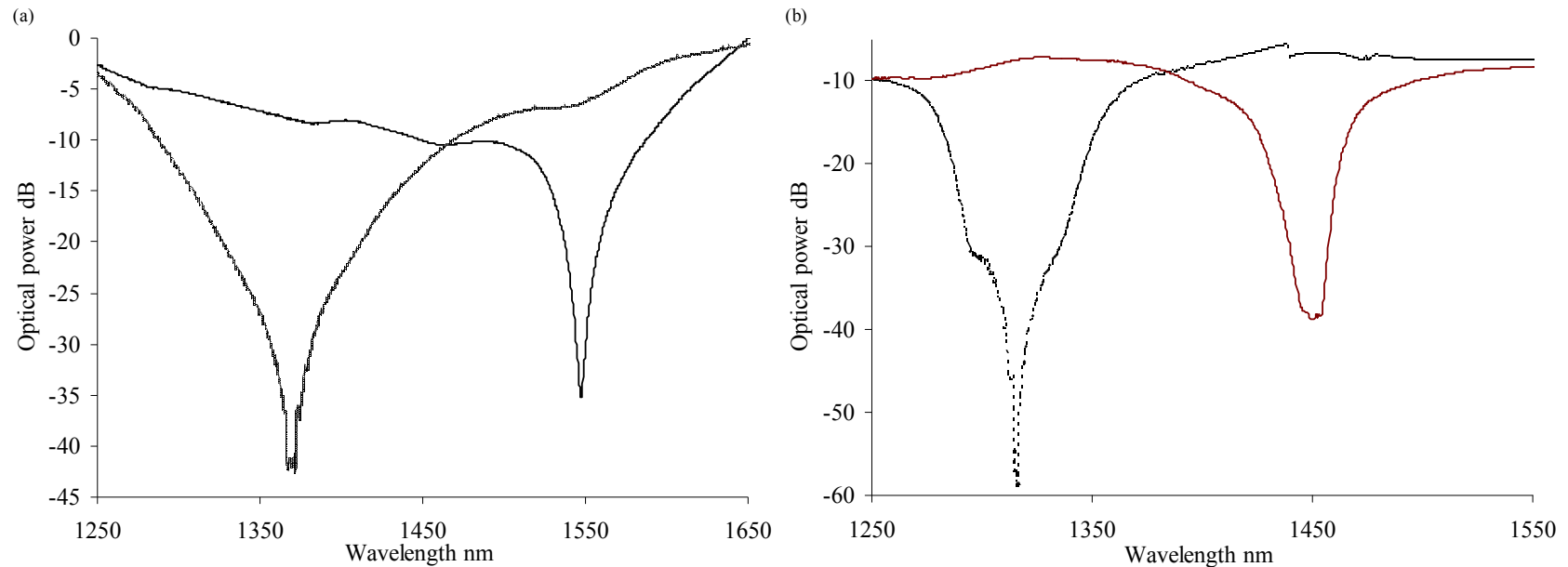

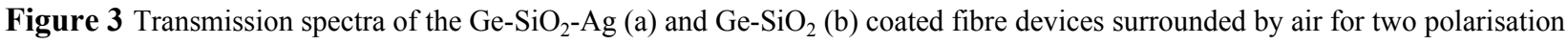
states of the illuminating light

It was also observed that polarisation sensitivity varied from device to device investigated, figure 4 and figure 5 show typical examples of the sensitivities of these devices
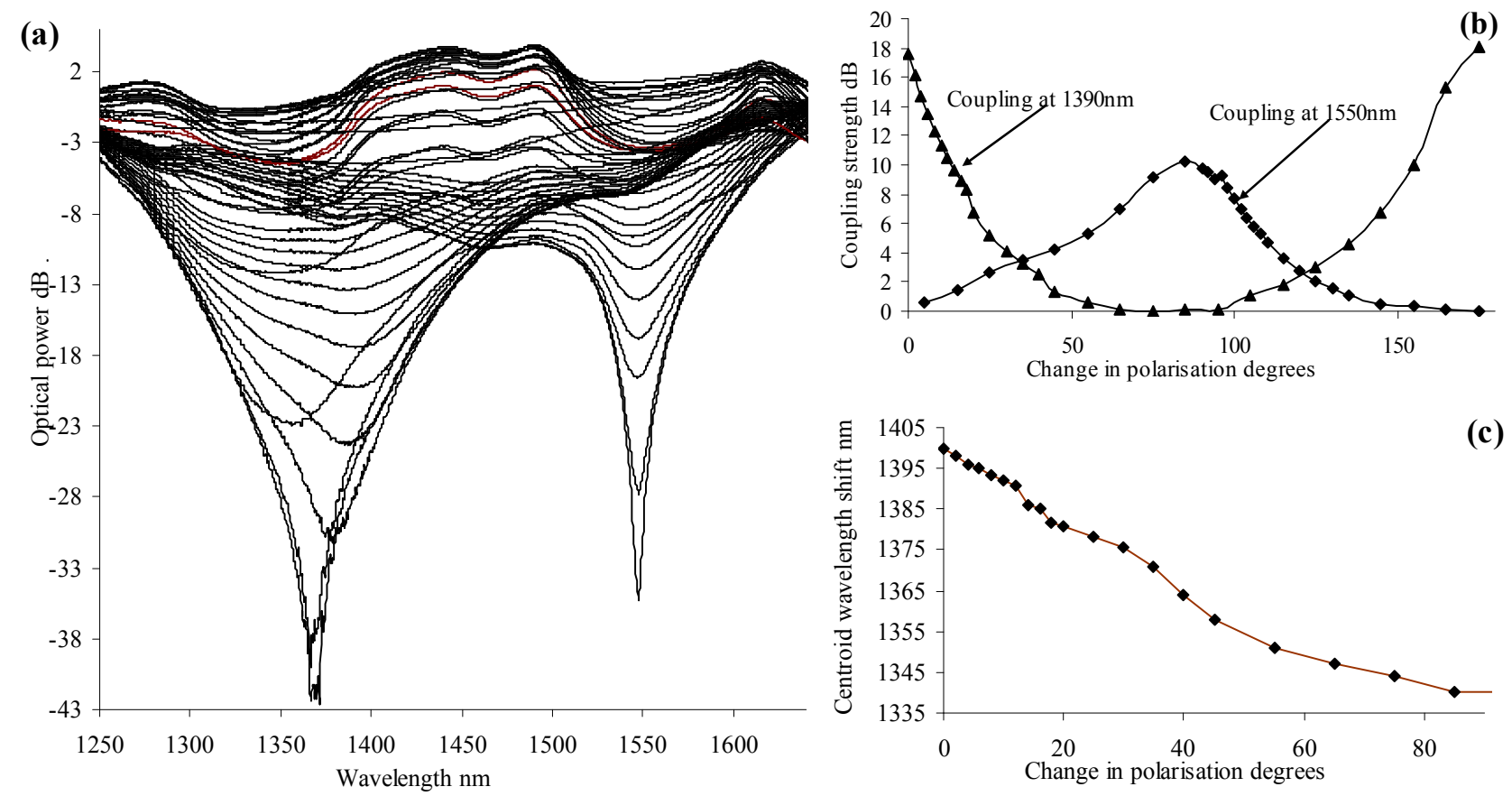

Figure 4 (a) Transmission spectra of the $\mathrm{Ge}_{-} \mathrm{SiO}_{2}-\mathrm{Ag}$ coated surface relief fibre device as a function of changing polarisation from maximum coupling in air. (b) The coupling efficiency of the resonances at $1390 \mathrm{~nm}$ and $1550 \mathrm{~nm}$ and (c) the wavelength shift of the coupling feature at $1390 \mathrm{~nm}$ as the azimuth of polarisation of the illuminating light is changed from the position providing maximum coupling. 

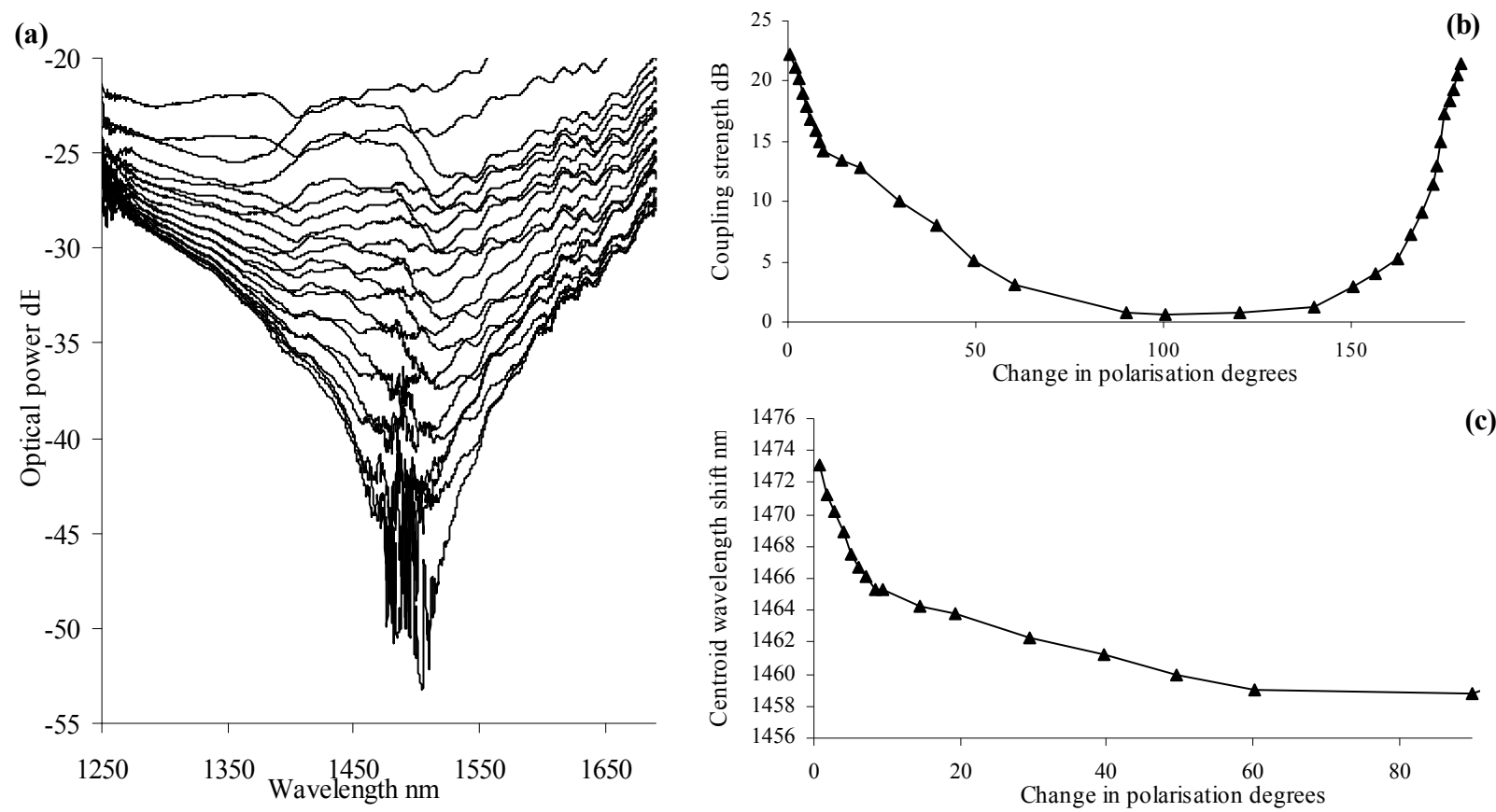

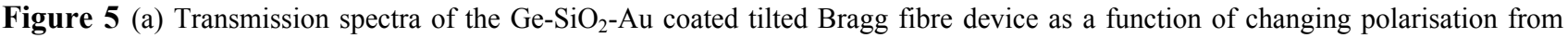
maximum coupling with a surrounding medium of 1.36. (b) The coupling efficiency of the resonance at $1480 \mathrm{~nm}$ and (c) the wavelength shift of the coupling feature at $1480 \mathrm{~nm}$ as the azimuth of polarisation of the illuminating light is changed from the position providing maximum coupling

We are mostly interested in the potential of these SPR devices for chemical and biochemical sensing applications using wavelength and intensity interrogation. Whilst it may seem that the polarisation sensitivity of theses SPR devices may be problematic, this can potentially be overcome by using polarisation maintaining fibre [13], or can be seen as another spectral property to be exploited. A detection scheme that does exploit this behaviour is angular interrogation [14]. Inspecting the transmission spectra in figures 2 and 5, there is no significant observed spectral feature associated with Bragg reflection from the gratings themselves. This is expected due to the fact their transmission profiles are very weak and that the interrogating light source is broadband and swamps the response.

\section{REFRACTIVE INDEX SENSITIVITY}

For refractive index sensitivity measurements the devices were placed in a V-groove and immersed in certified refractive index (CRI) liquids (supplied by Cargille laboratories Inc.) which have a quoted accuracy of \pm 0.0002 . The devices and $\mathrm{V}$-groove were carefully cleaned, washed in ethanol, then in deionised water and finally dried before immersion into the next CRI liquid. The V-groove was made in an aluminium plate, machined flat to minimise bending of the fibre. The plate was placed on an optical table, which acted as a heat sink to maintain a constant temperature. The V-groove was used in conjunction with the apparatus shown in figure 1. Figures 6 to 9 show some typical spectral index sensitivities measured for the devices. 

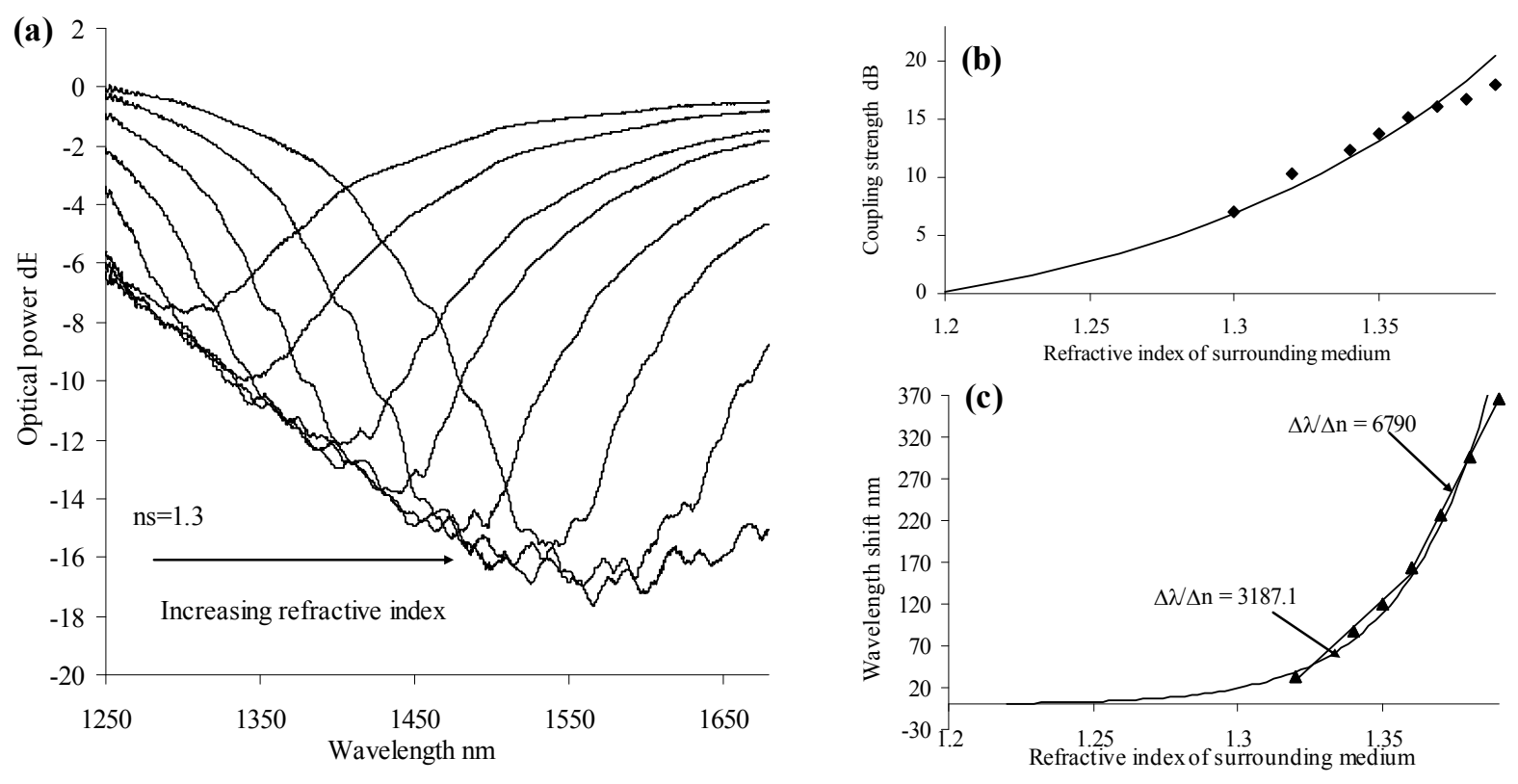

Figure 6. (a) Transmission spectra of the Ge coated surface relief device as a function of refractive index (polarisation of the illuminating light chosen to maximise coupling at index 1.3). (b) Coupling strength and (c) Wavelength shift of the resonance as a function of refractive index.

(a)

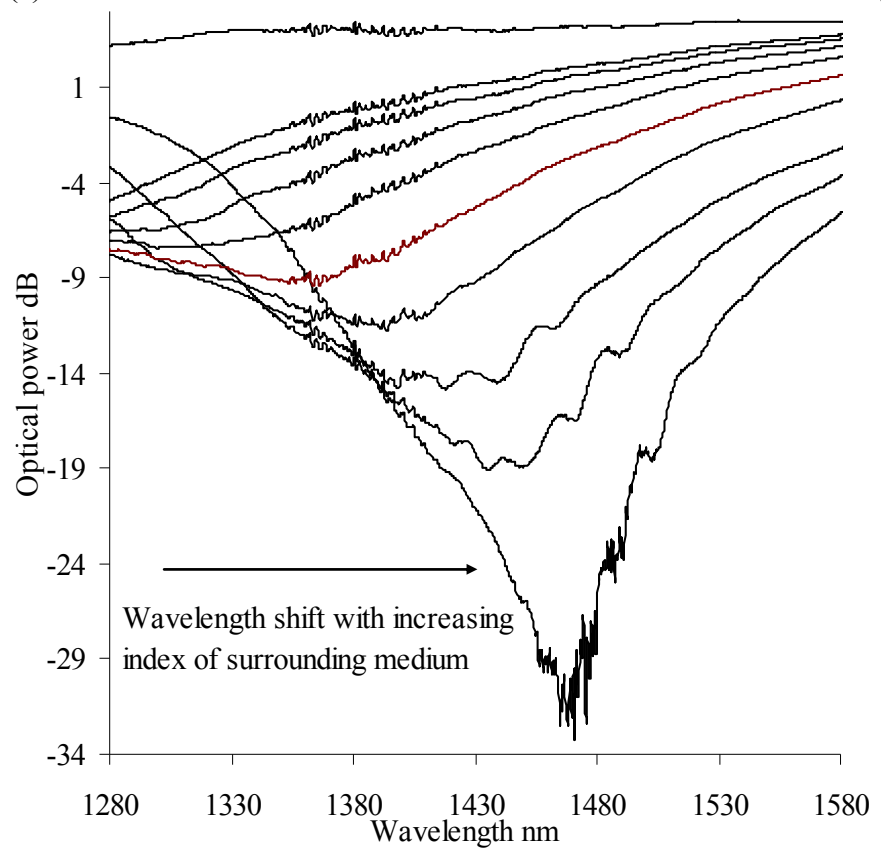

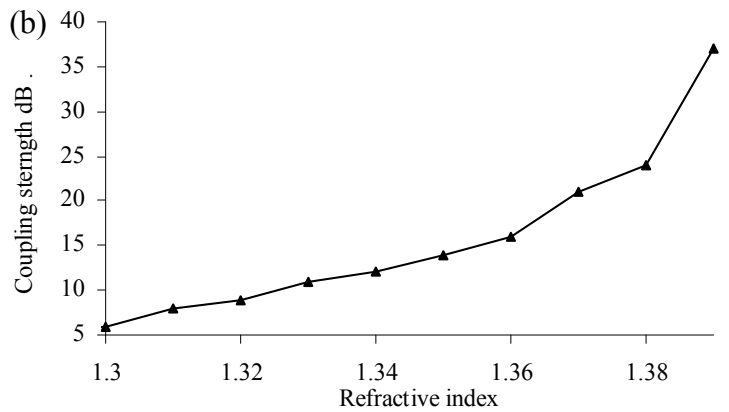

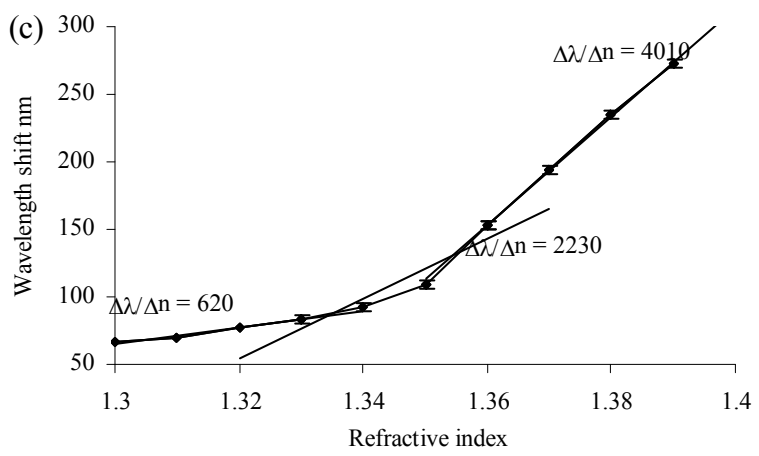

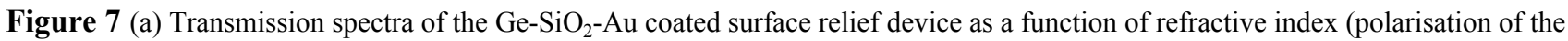
illuminating light chosen to maximise coupling at index 1.36). (b) Coupling strength and (c) Wavelength shift of the resonance as a function of refractive index. 

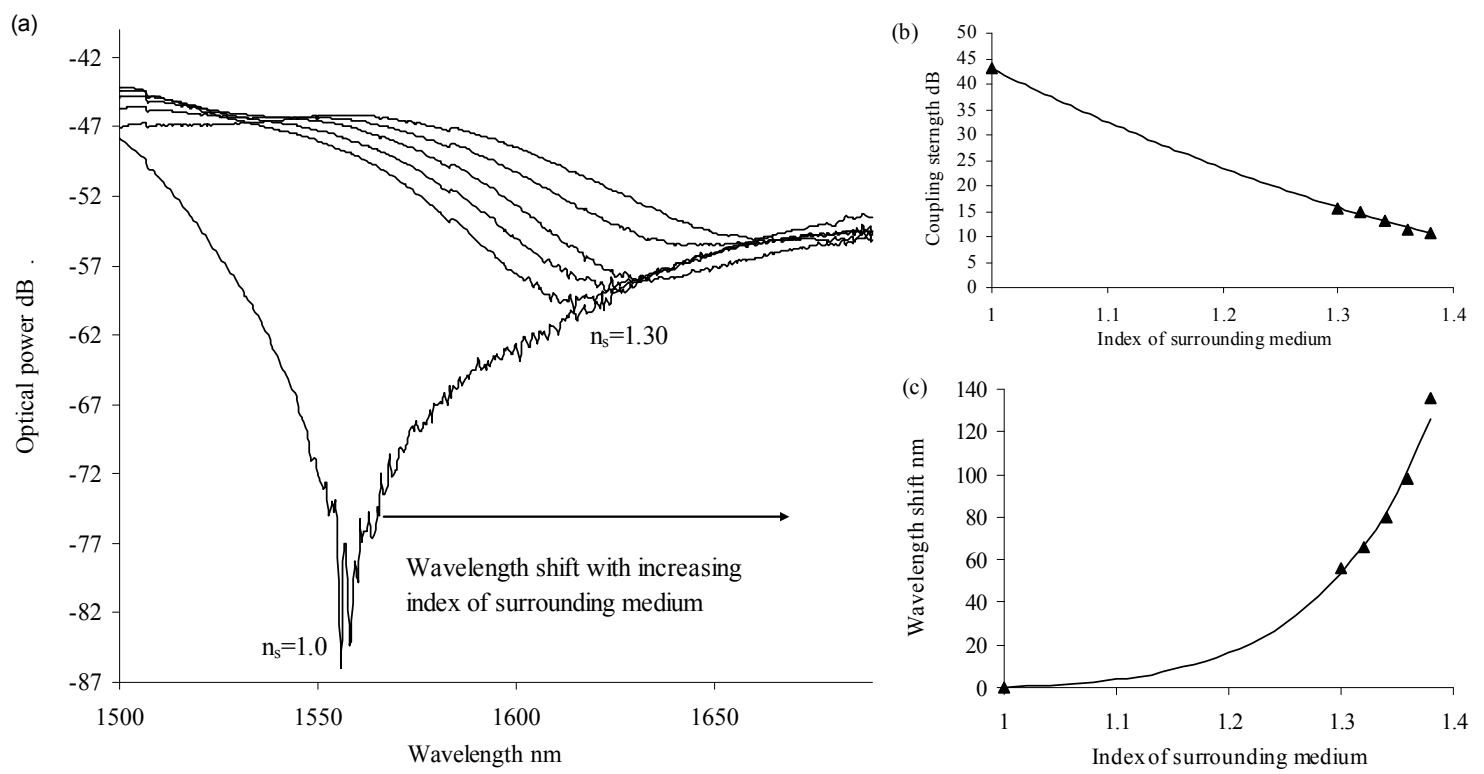

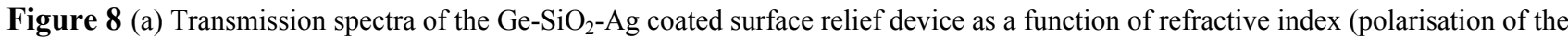
illuminating light chosen to maximise coupling at index 1.36). (b) Coupling strength and (c) Wavelength shift of the resonance as a function of refractive index.

(a)

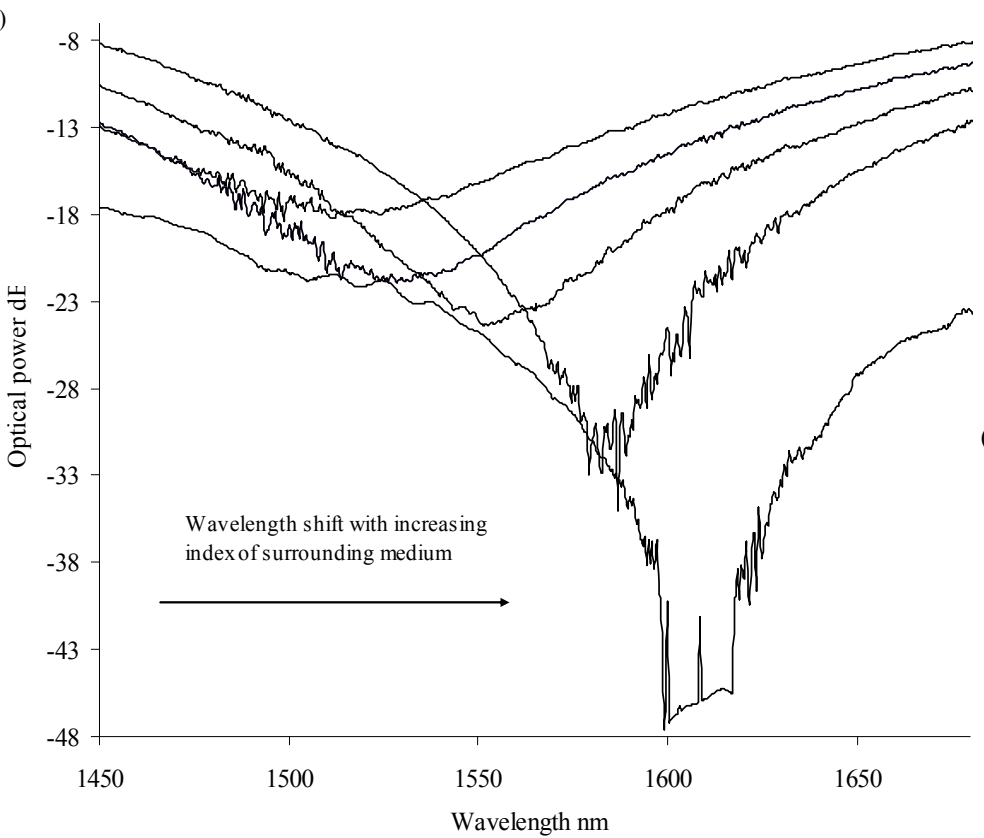

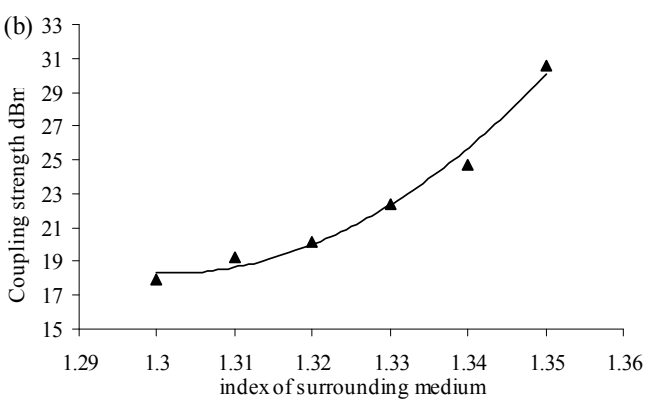

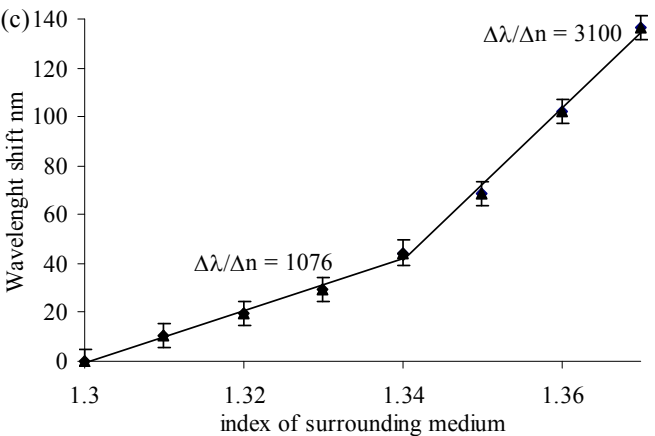

Figure 9 (a) Transmission spectra of the Ag coated tilted Bragg device (7 degrees) as a function of refractive index (polarisation of the illuminating light chosen to maximise coupling at index 1.36). (b) Coupling strength and (c) Wavelength shift of the resonance as a function of refractive index.

The highest wavelength spectral sensitivity to index was obtained with the Ge coating, which provided $\Delta \lambda / \Delta \mathrm{n}$ ranging from $3200 \mathrm{~nm}$ to $12500 \mathrm{~nm}$, see figure 6. Some the devices exhibited strong coupling in air such as the $\mathrm{Ge}^{-\mathrm{SiO}_{2}-\mathrm{Ag}}$ surface relief device which shows a decrease in coupling strength with increase in index, varying from $40 \mathrm{~dB}$ in air to $7 \mathrm{~dB}$ with a surrounding index of 1.39 and having a spectral sensitivity ranging from $710 \mathrm{~nm}$ to $1200 \mathrm{~nm}$ in the aqueous regime. The $\mathrm{Ge}_{-} \mathrm{SiO}_{2}-\mathrm{Au}$ surface relief device gave index sensitivities ranging from $2000 \mathrm{~nm}$ to $4500 \mathrm{~nm}$ with increasing 
coupling strength from no coupling to $37 \mathrm{~dB}$. Considering the low index regime ranging from 1 to 1.15 , the gold overlay fibre showed no coupling but the $\mathrm{Ge}_{-} \mathrm{SiO}_{2}-\mathrm{Ag}$ and $\mathrm{Ge}_{-} \mathrm{SiO}_{2}$ gave promising results with wavelength shifts of $90 \mathrm{~nm}$ and $40 \mathrm{~nm}$ along with $30 \mathrm{~dB}$ and $17 \mathrm{~dB}$ changes in optical coupling from index 1 (air) to 1.3 , respectively. The differently configured SPR devices yielded different responses for various index regimes results with some out-performing others for a given index range. This behaviour is expected due to the different dispersion relationships for each of the materials used in the coatings along with how the surface plasmons are supported by the different physical structures of the coatings.

To obtain an estimate of the spectral sensitivity of these SPR devices for low refractive indices from 1 to 1.1 we use the approach given in REF 15, which is to calculate the propagating modes of a D-shaped fibre with coating using a conformal mapping technique and then implement Fresnel's equations for a four layered system. The optical constants used in Fresnel's equations were estimated by an effective medium approximation, the Maxwell-Garnett theory [16], which yields an effective dielectric function as a function of the fractional volume of the metal/semi-conductor within an effective layer. In this work the effective change of the surrounding index is determined within a sensing volume, which is the area of the coating multiplied by the spatial extension of the evanescent field associated with the surface plasmon perpendicular to the coating's surface; a more detailed description is given in REF 6 and REF 11.

The experimental and theoretical results for wavelength and optical power spectral sensitivities are combined and shown in figures 10 and 11 . These results are compared to a long period grating with a period of $240 \mu \mathrm{m}$ and a length of $5 \mathrm{~cm}$ inscribed into standard single mode telecoms fibre. To attach any worth to these fibre sensors, their performance has to be compared to other sensor types in terms of wavelength shift (spectral sensitivity) and coupling strength variation (intensity sensitivity) as a function of index change. These results suggest the devices have potential in the low index regime and should demonstrate dramatic improvement in sensitivity/performance over LPG fibre devices in the aqueous index regime.

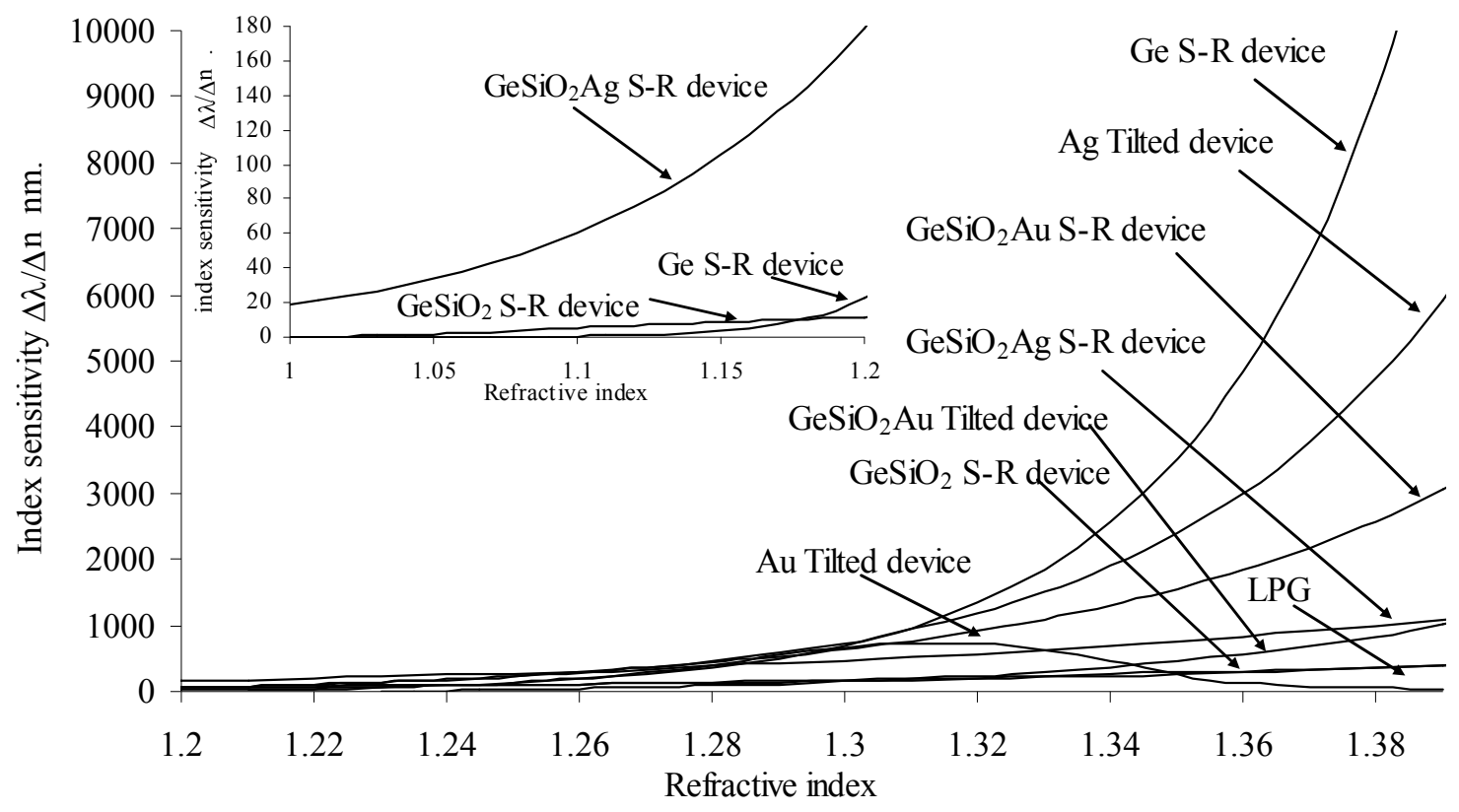

Figure 10 Simulated and experimental wavelength spectral sensitivity comparison of some of the coated fibre devices (Tilted devices; SPR fibre devices that have a tilted Bragg grating written into the core of the fibre, S-R devices; SPR fibre devices with a surface-relief corrugation of the fibre coatings) as a function of refractive index along with a long period grating (period $=240 \mu \mathrm{m}$, length $5 \mathrm{~cm})$. 


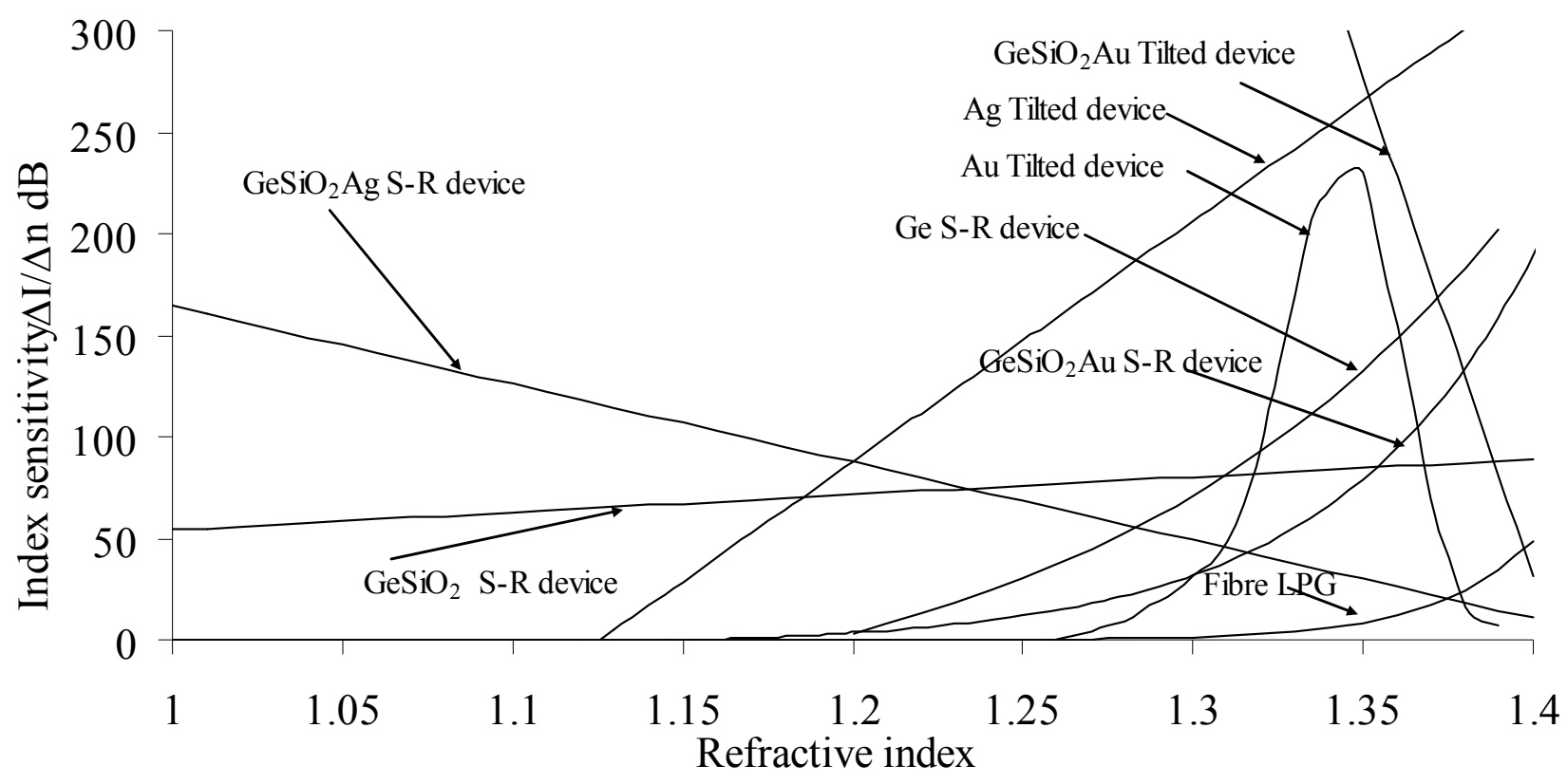

Figure 11 Simulated and experimental optical power sensitivity comparison of some of the coated fibre devices (Tilted devices; SPR fibre devices that have a tilted Bragg grating written into the core of the fibre, S-R devices; SPR fibre devices with a surface-relief corrugation of the fibre coatings) as a function of refractive index along with a long period grating (period $=240 \mu \mathrm{m}$, length $5 \mathrm{~cm}$ ).

\section{CONCLUSION}

We have investigated two types of surface plasmon resonance fibre devices with coupling mechanisms based upon either tilted fibre Bragg gratings or a surface relief structuring of the coating itself produced by ultra-violet exposure through a conventional fibre phase mask. The coatings used were both single and multi-layered thin films deposited on the flat side of a lapped D-shaped fibre. The various devices yielded different index sensitivities, with the single Ge coated device

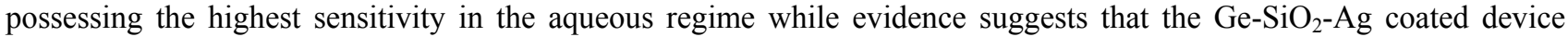
should be the most sensitive at low indices, both devices outperforming LPGs in these regimes. Some of the SPR devices have high coupling efficiency in excess of $40 \mathrm{~dB}$ in air and the $\mathrm{Ge}_{-} \mathrm{SiO}_{2}-\mathrm{Ag}$ coated device possessed an estimated index spectral sensitivity of $\Delta \lambda / \Delta \mathrm{n}=90 \mathrm{~nm}$ and a coupling strength sensitivity of $\Delta \mathrm{I} / \Delta \mathrm{n}=165 \mathrm{~dB}$ in the index range from 1 to 1.15. A device with a single layer of Ge demonstrated an index spectral sensitivity of $\Delta \lambda / \Delta \mathrm{n}=12500 \mathrm{~nm}$ over the index range of 1.36 to 1.39 with higher sensitivities obtained at higher indices.

\section{References}

[1] S. Vasilev et al, "Long-period refractive index fibre gratings: properties, applications and fabrication techniques", Proc. SPIE 4083, pp.212-223, 2000.

[2] R.P. Espindola, R. Windeler, A. Abramov, B. Eggleton, T. Strasser, D. DiGiovanni, "External refractive index insensitive air-clad long period fibre grating", Electron. Lett., vol.35, no.4, 327-328 1999.

[3] T. Allsop et al, " Detection of Organic Aromatic compounds in Paraffin by a long period fiber grating optical sensor with optimised sensitivity", Opt. Commun. ,vol 191, pp181,2001.

4 A. Dunn and R. Richards-Kortum, “Three-dimensional computation of light scattering from cells,” IEEE J. Sel. Top. Quantum Electron. 2, pp. 898-905, 1996.

[5] Joseph M. Schmitt and Gitesh Kumar, "Optical scattering properties of soft tissue: a discrete particle model”, Appl. Optics, Vol. 37, No. 13, pp.2788-2798, 1998 
[6] Sean D. Puckett and Gilbert E. Pacey, "Detection of water in jet fuel using layer-by-layer thin film coated long period grating sensor", Talanta, Vol. 78, Issue 1, pp.300-304, 2009

[7] S. Patskovsky et al, "Properties and sensing characteristics of surface plasmon resonance in infrared light", J. Opt. Soc. Am. A, Vol.20, No. 8, pp.1644-1650, 2003.

[8] "Surface Plasmons on smooth and Rough Surfaces and on Gratings", H. Raether, eds. (Academic, New York, 1997),

[9] T. Allsop et al, "Surface Plasmon Resonance Generation Utilising Gratings for Biochemical Sensing", OFS-18 Cancun Mexico, Biological and Medical Sensors, paper WA4, Oct 2006.

[10] T. Allsop et al, "Characterization of infrared surface plasmon resonances generated from fiber optical sensor utilizing tilted Bragg gratings", JOSA B, Vol. 25, No. 4, 2008

[11] A K. Sharma et al, "Influence of dopants on the performance of a fiber optic surface plasmon resonance sensor" Optic Comms Vol. 274, pp.320-326, 200

[12] M. Csete, et al, "Atomic force microscopical and surface plasmon resonance spectroscopical investigation of sub-micrometer metal gratings generated by UV laser-based two-beam interference in Au-Ag bimetallic layers", Applied Surface Science, Vol. 253, pp.7662-7671, 2007.

[13] M. Piliarik et al, "Surface plasmon resonance sensor based on a single-mode polarisation-maintaining optical fiber", Sensors and Actuators B, Vol. 90, pp.236-242, 2004

[14] E.M. Yeatman, "Resolution and sensitivity in surface plasmon microscopy and sensing", Biosensors and Bioelect., Vol. 11, No. 6, pp.635-649, 1996.

[15] T. Allsop et al, "The exploitation of multilayer coatings for infra-red surface plasmon resonance fibre sensors", Appl. Optics., Vol.48, pp. 276-286, 2009

[16] D. E. Aspnes, J. B. Theeten, F. Hottier, " Investigation of effective-medium models of microscopic surface roughness by spectroscopic ellipsometry,” Phys. Rev. B 20, No. 8, pp.3292 - 3302, 1979. 